\title{
Histological Study and LYVE-1 Immunolocalization of Mouse Mesenteric Lymph Nodes with "In Vivo Cryotechnique"
}

\author{
Yuqin Bai $^{1, *}$, Bao $\mathrm{Wu}^{1}$, Nobuo Terada ${ }^{1}$, Nobuhiko Ohno ${ }^{1}$, Sei Saitoh ${ }^{1}$, Yurika Saitoh ${ }^{1}$ \\ and Shinichi Ohno ${ }^{1}$ \\ ${ }^{1}$ Department of Anatomy and Molecular Histology, Interdisciplinary Graduate School of Medicine and Engineering, University of \\ Yamanashi, 1110 Shimokato, Chuo-city, Yamanashi 409-3898, Japan
}

Received January 7, 2011; accepted March 8, 2011; published online April 21, 2011

\begin{abstract}
The "in vivo cryotechnique" (IVCT) is a powerful tool to directly freeze living animal organs in order to maintain biological components in frozen tissues, reflecting their native states. In this study, mesenteric lymph nodes of living mice were directly frozen with IVCT, and we did morphological studies and immunohistochemical analyses on a hyaluronic acid receptor, LYVE-1. In lymph nodes, widely open lymphatic sinuses were observed, and many lymphocytes adhered to inner endothelial cells along subcapsular sinuses. The LYVE-1 was clearly immunolocalized at inner endothelial cells of subcapsular sinuses, as well as those of medullary sinuses. Conventional pre-embedding electron microscopy also showed LYVE-1 immunolocalization along both the apical and basal sides of cell membranes of inner endothelial cells. By triple-immunostaining for LYVE-1, smooth muscle actin, and type IV collagen, the LYVE-1 was immunolocalized only in the inner endothelial cells, but not in outer ones which were surrounded by collagen matrix and smooth muscle cells. Thus, the functional morphology of lymph nodes in vivo was demonstrated and LYVE-1 immunolocalization in inner endothelial cells of subcapsular sinuses suggests hyaluronic acid incorporation into lymph node parenchyma.
\end{abstract}

Key words: immunohistochemistry, in vivo cryotechnique, LYVE-1, mesenteric lymph node

\section{Introduction}

Lymph nodes are peripheral lymphatic organs connecting afferent lymphatic vessels to efferent ones via subcapsular, intermediate, and medullary sinuses. Some markers specific for lymphatic vessels are lymphatic vessel endothelial hyaluronan receptor-1 (LYVE-1) [2, 25], vascular endothelial growth factor receptor-3 (VEGFR-3) [13], prox $1[26,46]$, and podoplanin [3, 45]. LYVE-1 is a $322-$ amino acid transmembranous glycoprotein homologous to

Correspondence to: Shinichi Ohno, M.D., Ph.D., Department of Anatomy and Molecular Histology, Interdisciplinary Graduate School of Medicine and Engineering, University of Yamanashi, 1110, Shimokato, Chuo-city, Yamanashi 409-3898, Japan.

E-mail: sohno@yamanashi.ac.jp

*Dr. Y. Bai was a research fellow from the Department of Pathology, Faculty of Medicine, Chifeng University, Inner Mongolia 024000, China, while this work was in progress at the University of Yamanashi. an inflammatory leukocyte homing receptor, CD44, and both of them are receptors for hyaluronic acid [8, 9, 29]. In lymphatic sinuses of rat lymph nodes, LYVE-1 was reported to be localized in endothelial and reticular cells of medullary sinuses [25]. Recently, it was also reported that a type of macrophage expressed LYVE-1 in some lymphatic vessels [28]. Therefore, it is of interest to examine the LYVE-1 immunolocalization in lymph nodes because they have specific lymph sinuses surrounded by endothelial cells which connect to the lymphatic vessels.

To clarify the morphological states of functioning animal organs, we have already proposed that the "in vivo cryotechnique" (IVCT) is a powerful tool by which living animal organs are directly frozen in vivo [23, 38]. IVCT can prevent morphological artifacts of cells and tissues caused by tissue-resection and immersion- or perfusion-fixation [1, $21,31]$. It is also possible to examine immuolocalization of soluble proteins, as well as small amino acids, with high 
immunoreactivity, reflecting their original localization $[15$, $21,34,39,48]$. In this study, we performed morphofunctional analyses of mouse mesenteric lymph nodes under normal blood circulation prepared with IVCT, and examined immunolocalization in situ of LYVE-1, type IV collagen and smooth muscle actin in the lymph nodes.

\section{Materials and Methods}

\section{Tissue preparation using the "in vivo crotechnique" (IVCT) or conventional methods}

All animal experiments were performed in accordance with guidelines by the Animal Care and Use Committee, University of Yamanashi. A total of 15 adult C57BL/6J male mice, weighing 20-30 g, were prepared by the following different methods. (i) In vivo cryotechnique (IVCT) (Fig. 1): Under pentobarbital anesthesia, abdominal cavities of 9 mice were opened and mesenteric lymph nodes were carefully exposed (Fig. 1a). Isopentane-propane cryogen $\left(-193^{\circ} \mathrm{C}\right)$ cooled in liquid nitrogen was directly poured over mesenteric lymph nodes (Fig. 1b) while their hearts were beating, in a similar way to that for living mouse livers [21]. Then, the frozen lymph nodes were removed with a dental electric drill in liquid nitrogen (Fig. 1c), and processed for freeze-substitution (FS) fixation. Briefly, the frozen specimens were freeze-substituted in acetone containing $2 \%$ paraformaldehyde (PFA) at $-80^{\circ} \mathrm{C}$ in dry ice-acetone for $48 \mathrm{hr}$, and gradually warmed up to room temperature (RT). (ii) Immersion-fixation and alcoholdehydration (IM-DH): Mesenteric lymph nodes of 3 mice were surgically resected and immersed into $2 \%$ PFA in $0.1 \mathrm{M}$ phosphate-buffer solution (PB; $\mathrm{pH} 7.4)$ for $2 \mathrm{hr}$ at RT. (iii) Perfusion-fixation and alcohol-dehydration (PF$\mathrm{DH})$ : Three mice were transcardially perfused with $2 \%$ PFA in $0.1 \mathrm{M} \mathrm{PB}$, and their mesenteric lymph nodes were

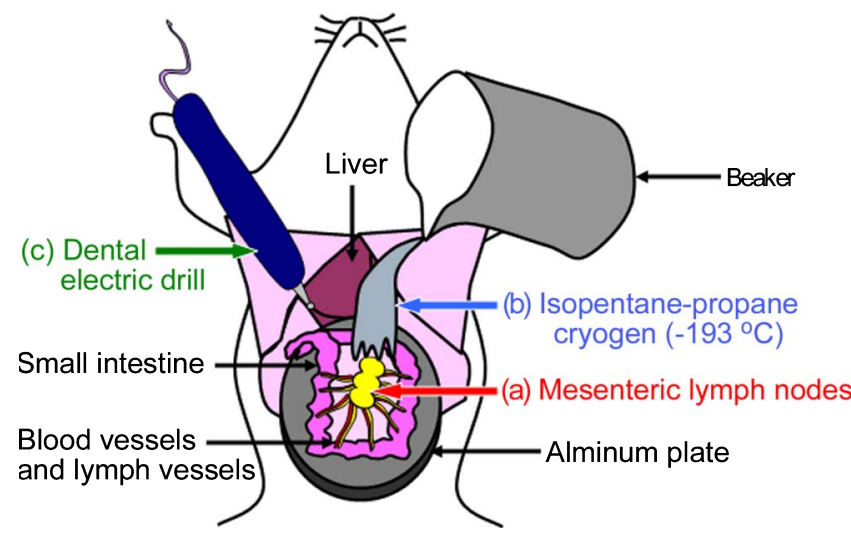

Fig. 1. Schematic representation of the "in vivo cryotechnique" (IVCT) for mouse mesenteric lymph nodes. Under anesthesia, mesenteric lymph nodes were exposed (a), and isopentane-propane cryogen $\left(-193^{\circ} \mathrm{C}\right)$ was directly poured over them $(\mathbf{b})$. Then, the frozen lymph nodes were taken out with a dental electric drill in liquid nitrogen (c), and processed for freeze-substitution (FS) fixation. resected and additionally immersed in the same fixative for $2 \mathrm{hr}$. The specimens prepared by the latter two methods, (ii) and (iii), were processed for common alcohol-dehydration. Fixed lymph nodes were routinely embedded in either paraffin wax or $30 \%$ sucrose for cryosections, as described previously [18, 37].

\section{Immunostaining for light microscopy}

Paraffin sections (2-4 $\mu \mathrm{m}$ thick) or cryosections (6 $\mu \mathrm{m}$ thick) were cut, and attached to Matsunami Adhesive Slide (MAS)-coated glass slides (Matsunami Glass, Osaka, Japan). Some deparaffinized sections were stained with hematoxylin-eosin (HE) to obtain morphological findings. The other deparaffinized sections or cryosections were rehydrated in phosphate-buffered saline (PBS) and immunostained with various antibodies, as previously described [22]. Briefly, all serial sections for the immunoperoxidase staining were first blocked with $1 \%$ hydrogen peroxide in PBS, and incubated in a blocking solution, PBS containing $5 \%$ normal goat serum (NGS) (Vector Laboratories; Burlingame, CA, USA) for $1 \mathrm{hr}$. They were immunostained with rabbit polyclonal anti-LYVE-1 (Abcam; Cambridge, UK) in PBS containing $5 \% \mathrm{NGS}$ at $4^{\circ} \mathrm{C}$ overnight. The immunostained sections were then incubated in biotin-conjugated goat anti-rabbit IgG (Vector Lab.) at RT for $1 \mathrm{hr}$. The immunoreaction products were visualized with Vectastain ${ }^{\circledR}$ avidin-biotin complex (ABC) reagent (Vector Lab.) and a metal-enhanced diaminobenzidine (DAB) substrate kit (Pierce; Rockford, IL, USA), and additionally fixed with $0.04 \%$ osmium tetroxide $\left(\mathrm{O}_{\mathrm{S}} \mathrm{O}_{4}\right)$ solution, as described before [21]. All immunostained paraffin sections were counterstained with methylgreen, embedded in glycerol, and observed in a light microscope (BX-61; Olympus, Tokyo, Japan).

For triple-immunostaining for LYVE-1, type IV collagen and smooth muscle actin, some cryosections were blocked with 5\% gelatin in PBS for $1 \mathrm{hr}$, and immunostained with a rat monoclonal anti-type IV collagen $\alpha 2$-chain (H22) antibody [30] at $4^{\circ} \mathrm{C}$ overnight. After washing in PBS, they were incubated with a biotinylated donkey anti-rat IgG antibody (Jackson Immunoresearch Laboratories; West Grove, PA, USA), a rabbit polyclonal anti-LYVE-1 antibody and a mouse anti-smooth muscle actin antibody (Thermo Fisher Scientific; Fremont, CA, USA) at RT for $1 \mathrm{hr}$. Next, they were incubated with Alexa Fluor 546-conjugated streptavidin, an Alexa Fluor 488-conjugated donkey anti-rabbit IgG antibody and an Alexa Fluor 633-conjugated goat antimouse IgG antibody (Invitrogen; Carlsbad, CA, USA) at RT for $1 \mathrm{hr}$. The immunostained sections were mounted with Vectashield (Vector Lab.), and observed under a confocal laser scanning microscope (FV 1000: Olympus; Tokyo, Japan).

\section{Pre-embedding immunostaining for electron microscopy}

Some parts of lymph node specimens prepared by perfusion-fixation were infiltrated in PBS containing 30\% sucrose [44], and $6 \mu \mathrm{m}$ cryosections were cut. They were 
immunostained for LYVE-1 with the common immunoperoxidase-DAB method as reported before [40, 41]. They were routinely embedded in epoxy resin by the inverted gelatin capsule method, and ultrathin sections were cut with an ultramicrotome. They were briefly stained with uranyl acetate alone and observed in a transmission electron microscope (H-7500: Hitachi; Tokyo, Japan).

\section{Results}

\section{Histological findings of lymph node tissues}

First, we obtained some histological findings with hematoxylin-eosin (HE) staining of mesenteric lymph nodes prepared by IVCT-FS (Fig. $2 \mathrm{a}-\mathrm{d}$ ). In the previous study, the tissue areas with hardly visible ice crystals were approximately 200-300 $\mu \mathrm{m}$, depending on the distance of the tissues from the organ surface [22]. In the present study, the areas marked with dotted lines were showing better morphology without tissue damage (Fig. 2b). Their surface structures were thin layers of dense connective tissues which form obvious capsules of lymph nodes ( $\mathrm{Cp}$ in Fig. 2a-c).
The capsules contained several afferent lymphatic vessels (ALV in Fig. 2a). Parenchyma of lymph nodes was divided into two common zones: a cortex (Co in Fig. 2a, b) and medulla (Me in Fig. 2a). The cortex consisted of an outer cortex (OC in Fig. 2a) with numerous lymphatic nodules (LyN in Fig. 2a) and an inner cortex (IC in Fig. 2a) with lymphocyte accumulation extending into the medulla (Me in Fig. 2a). Between the capsule of dense connective tissues and parenchyma, widely open subcapsular sinuses (SS in Fig. 2a, b) were observed with IVCT-FS, connecting to medullary sinuses (MeS in Fig. 2a, d) through intermediate sinuses (IS in Fig. 2a, c). Stripy appearance seen in the deep sinuses was because of freezing lymph fluid alone (Fig. 2c, d), as compared to the surface sinus (Fig. 2b). Many small lymphocytes with chromatin-condensed nuclei were localized in the lymphatic sinuses. Some lymphocytes adhered to inner endothelial cells along subcapsular sinuses (arrowheads in Fig. 2b). Thus, naturally open spaces of lymphatic sinuses and the in situ location of flowing lymphocytes were well retained with IVCT, probably showing their original living states.

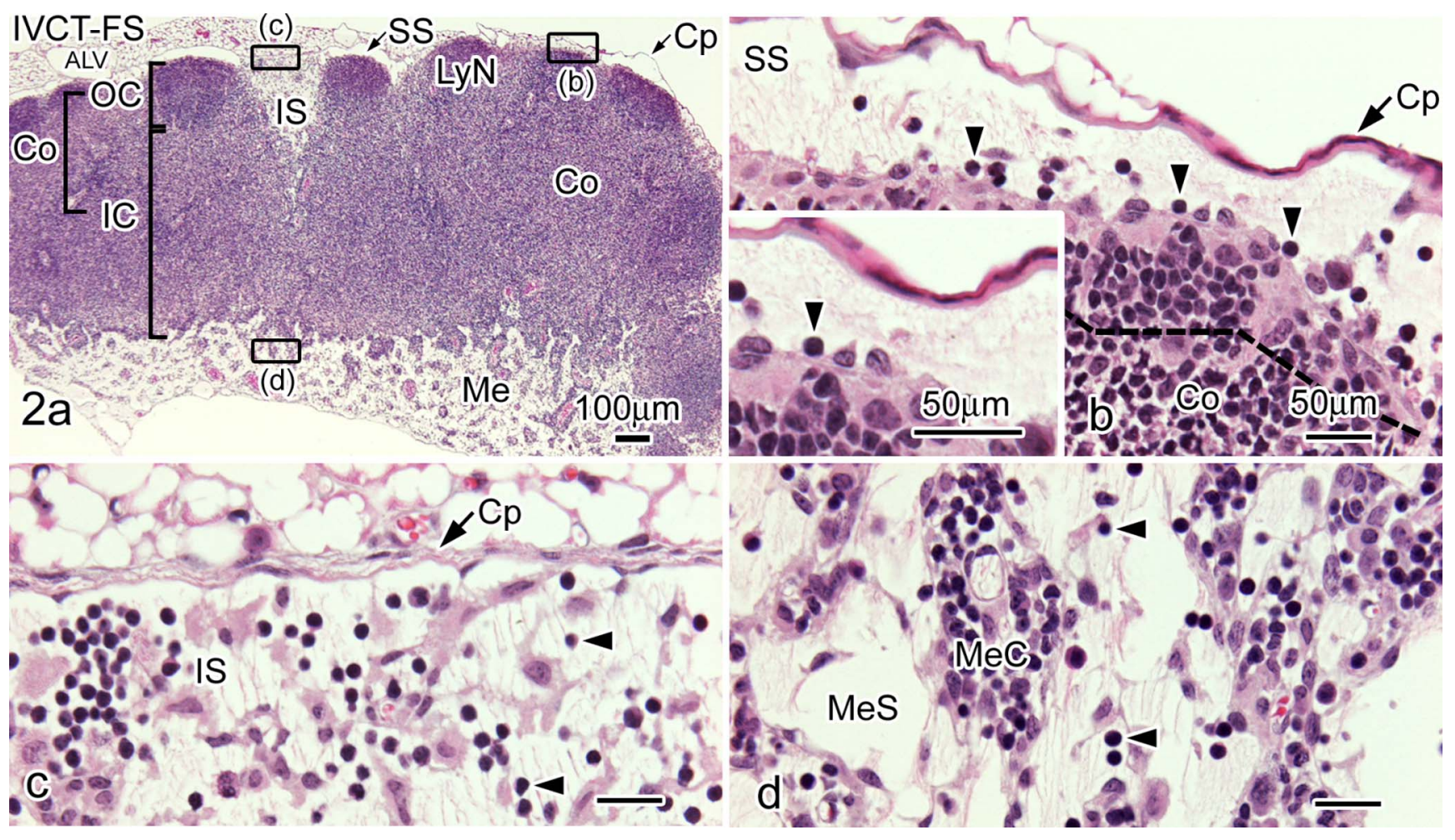

Fig. 2. Light micrographs of hematoxylin-eosin (HE) staining for paraffin sections of mesenteric lymph nodes prepared by IVCT followed by FS fixation. (a) Lower magnified view of a lymph node showing wide areas from the cortex (Co) to medulla (Me). In the cortex, outer (OC) and inner (IC) cortices are observed. Cp, capsule; ALV, afferent lymphatic vessel; IS, intermediate sinus; LyN, lymphatic nodule; SS, subcapsular sinus. The deep areas of dotted lines in this lymph node are showing tissue damage caused by ice crystal formation (b). The panels (b), (c) and (d) are higher magnified views of three parts shown as rectangles in (a). (b) Space of subcapsular sinus (SS) is opening, and the outside is surrounded by a capsule (Cp). Small lymphocytes with dense chromatin in nuclei are localized along inner endothelial cells of SS (arrowheads in b). (c) An intermediate sinus (IS) is connecting from SS. (d) Medullary cords (MeC) and open medullary sinuses (MeS) are clearly observed in the medulla of lymph nodes in vivo. Lymphocytes are observed in intermediate and medullary sinuses (arrowheads in $\mathbf{c}, \mathbf{d})$. Bars $=100 \mu \mathrm{m}(\mathbf{a})$, $50 \mu \mathrm{m}(\mathbf{b}-\mathbf{d})$. 

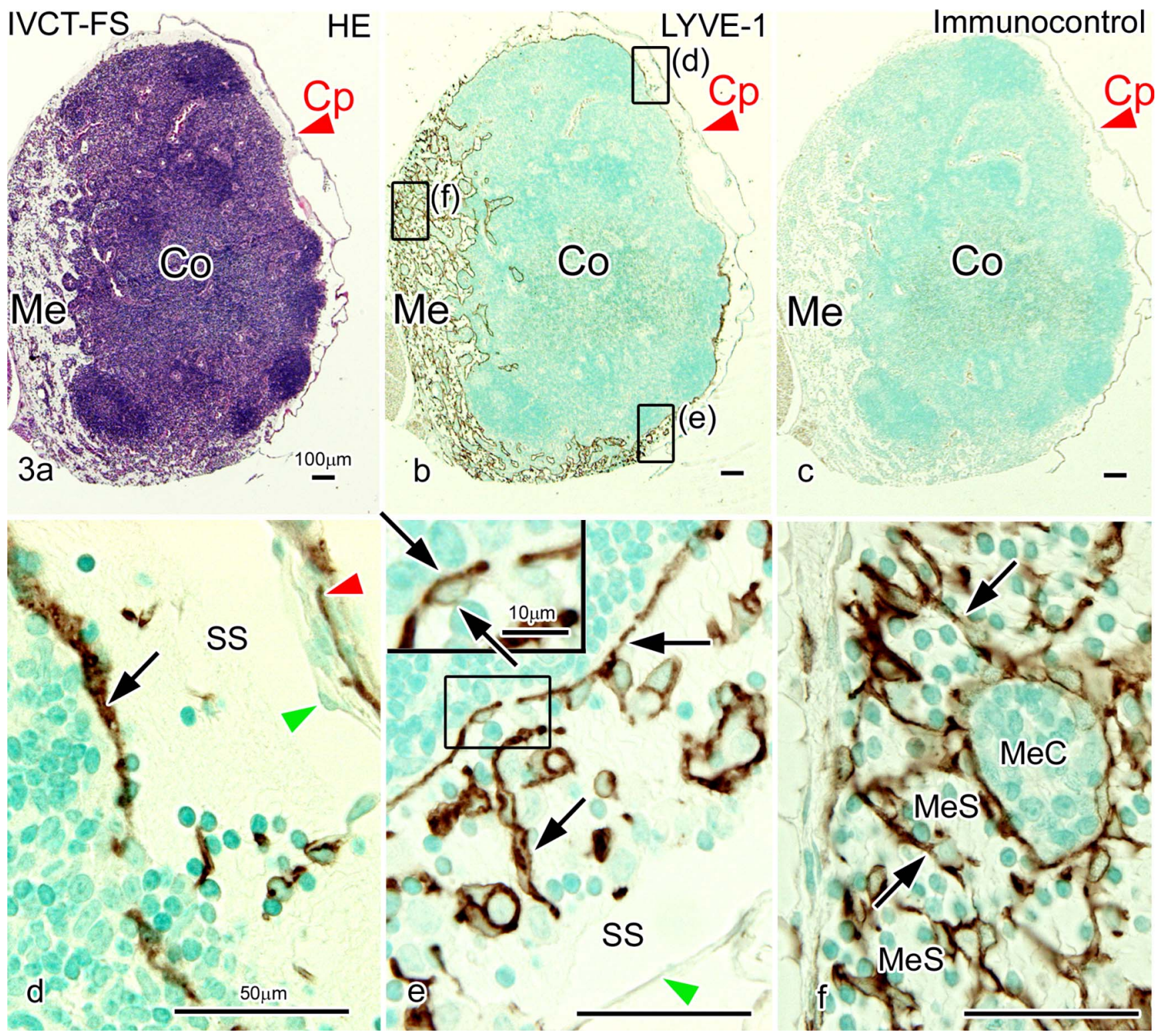

Fig. 3. Serial paraffin sections of a mesenteric lymph node prepared by IVCT-FS method stained with hemtoxylin-eosin (HE) (a), immunostained for LYVE-1 (b) or immunocontrol without the primary anti-LYVE-1 antibody (c). Panels (d), (e) and (f) show higher magnified views of three parts shown as rectangles in (b). Inset in (e) is highly magnified view of inner endothelia cells shown by the square. The LYVE-1 is immunolocalized in both luminal and basal cell membranes of inner endothelial cells (arrows in d, e), but not in outer endothelial cells (green arrowheads in d, e) of the subcapsular sinus (SS). LYVE-1 immunopositive cells in capsules (red arrowhead in d) are apparently different from the endothelium. In medullary sinuses (MeS in f), LYVE-1 (arrows in f) is immunolocalized in endothelial and reticular cells. Co, Cortex; Cp, Capsule; Me, Medulla; MeC, Medullary cord. Bars=100 $\mu \mathrm{m}(\mathbf{a}-\mathbf{c}), 50 \mu \mathrm{m}(\mathbf{d}-\mathbf{f}), 10 \mu \mathrm{m}$ (inset of e).

\section{Immunolocalization of LYVE-1 in lymphatic sinuses}

With IVCT-FS, it was easy to determine the immunolocalization of LYVE-1, because spaces of lymphatic sinuses were well maintained (Fig. 3), generally reflecting their living states. The LYVE-1 was immunolocalized along the inner side of subcapsular sinuses (arrows in Fig. $3 \mathrm{~d}$, e), showing inner endothelial cells and reticular cells. Along the outer side of subcapsular sinuses, LYVE-1 was immunolocalized in some cells among connective tissues of capsules (red arrowhead in Fig. 3d), but not in endothelial cells (green arrowhead in Fig. 3d, e), which might be a type of macrophage $[5,32]$. In medullary sinuses, LYVE1 was immunolocalized in endothelial and reticular cells (Fig. 3f). These findings indicate that the expression and localization of LYVE-1 are different among endothelial cells of the subcapsular sinuses.

\section{LYVE-1 immunolocalization in afferent lymphatic vessels, subcapsular sinuses and intermediate sinuses}

Next, the LYVE-1 immunolocalization was examined 

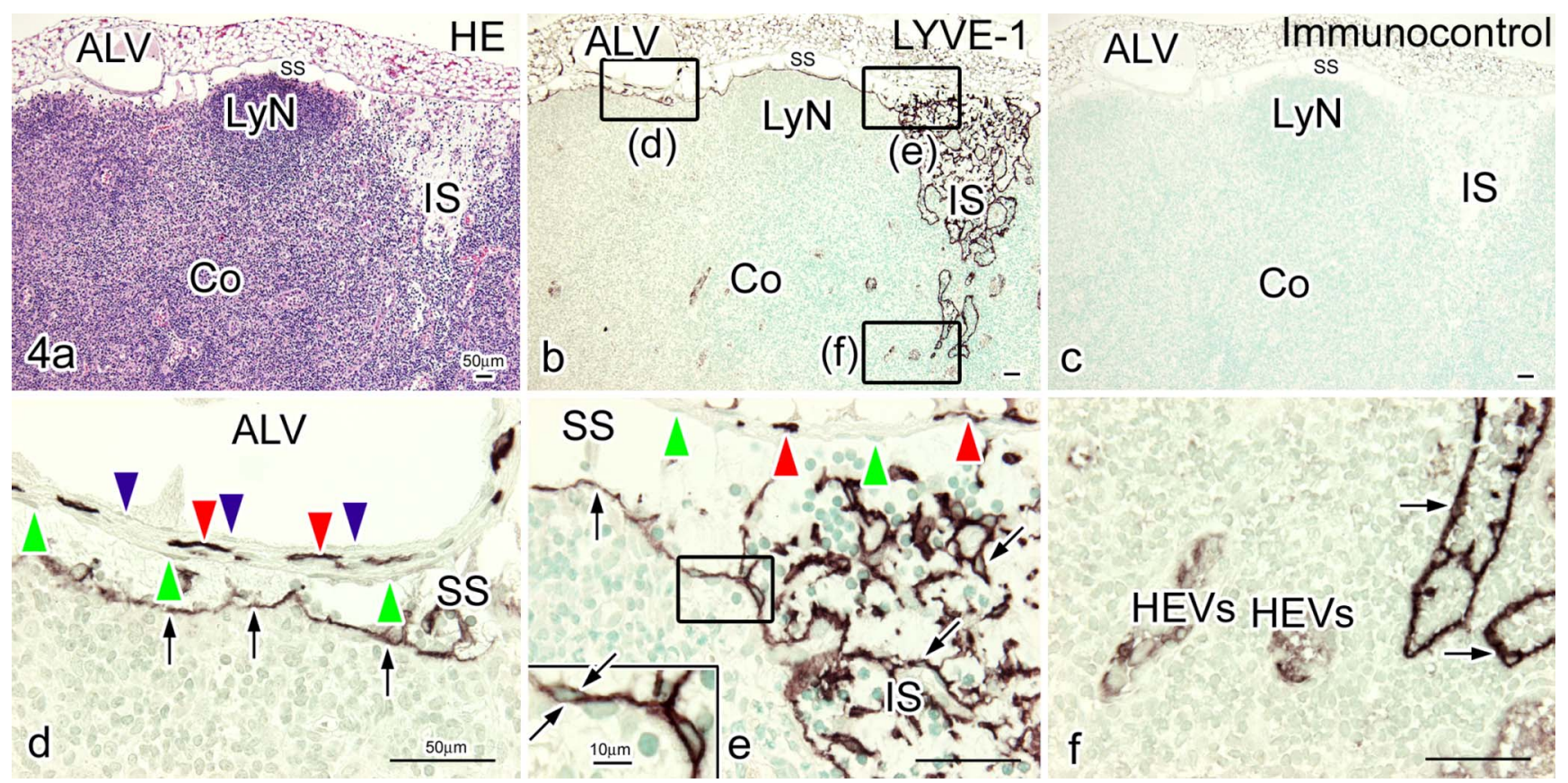

Fig. 4. Serial paraffin sections of a mesenteric lymph node prepared by IVCT-FS method, stained with hemtoxylin-eosin (HE; a), and immunostained for LYVE-1 (b, d, e, f) or immunocontrol without the primary anti-LYVE-1 antibody (c). The figure (d-f) shows higher magnified views of the parts shown as rectangles in (b). Inset in (e) is a highly magnified view of inner endothelial cells shown by the square. The LYVE1 is not immunolocalized in endothelial cells of an afferent lymphatic vessel (ALV; blue arrowheads in $\mathbf{d}$ ). In the subcapsular sinus (SS in $\mathbf{d}, \mathbf{e}$ ) facing the ALV and intermediate sinus (IS, e), LYVE-1-immunopositive cells are observed in the capsular stroma (red arrowheads in $\mathbf{d}, \mathbf{e}$ ) and reticular cells (black arrows in e) or both luminal and cell membranes of inner endothelial cells (black arrows in d-f), but not in outer endothelial cells (green arrowheads in $\mathbf{d}, \mathbf{e}$ ). The LYVE-1 is also immunolocalized in endothelial cells of high endothelial venules (HEVs in $\mathbf{f}$ ). Co, Cortex; Cp, Capsule; LyN, Lymphatic nodule. Bars $=50 \mu \mathrm{m}(\mathbf{a}-\mathbf{d}), 10 \mu \mathrm{m}$ (inset of e).

from afferent lymphatic vessels into the mesenteric lymph nodes (Fig. 4). Along the afferent lymphatic vessel (ALV in Fig. 4), LYVE-1-immunopositive cells were observed in connective tissues of capsules (red arrowheads, Fig. 4d), but not in endothelial cells of ALV (blue arrowheads, Fig. 4d). Outer endothelial cells were LYVE-1-immunonegative along the subcapsular sinuses and intermediate sinuses, but LYVE-1 immunolocalization was seen at cell membranes of the inner endothelial cells (Fig. 4e, inset) and reticular cells, as described in the previous section. Thus, it was confirmed that the inner endothelial cells of subcapsular sinuses are initial portions for the appearance of LYVE-1 in lymph nodes. LYVE-1 immunolocalization at high endothelial venules (HEVs) was similar to that in the previous studies (Fig. 4f) [27].

\section{LYVE-1 immunoreactivity of lymph nodes with various preparation methods}

The LYVE-1 immunoreactivity was compared among various preparation methods (Fig. 5), because the cryotechniques such as IVCT and quick-freezing of fresh tissues (FQF) are better approaches to obtain strong immunoreactivity against some antibodies $[19,20]$, but translocation of soluble proteins was easily induced by ischemia, anoxia, and mechanical stress of resected tissues with FQF $[1,15]$.
The LYVE-1 immunoreactivity was stronger in tissues with IVCT-FS (Fig. 5a-c), compared to the conventional chemical fixation followed by alcohol-dehydration (Fig. 5di). By different antibody dilutions of 1:300 and 1:7500, the LYVE-1 immunoreactivity was clearly observed along cell membranes and basal sides of the inner endothelial cells, reticular cells or in some cells of the capsule (Fig. 5b, c). In addition, the immunolocalizations of LYVE-1 are interrupted in the inner endothelial cells (inset of Fig. 5c), as reported before [28]. Therefore, it was beneficial to use a lymphatic vessel marker, LYVE-1, in the IVCT-FS samples of lymph nodes to reflect the native states of subcapsular and intermediate sinuses.

\section{Immunoelectron microscopy of LYVE-1 in lymph nodes}

To determine more precise immunolocalization of LYVE-1 in endothelial cells along subcapsular sinuses, preembedding immunoelectron microscopy was performed for the perfusion-fixed mouse mesenteric lymph nodes (Fig. 5j, $\mathrm{k})$. Immunoreaction products were observed to be along cell membranes of inner endothelial cells in subcapsular sinuses (black arrows, Fig. 5j, k). They were localized not only at the apical sides, but also the basal sides of the endothelial cells, as reported before [30]. In addition, the immunolocalization of LYVE-1 was strongly observed at the luminal 
HE

LYVE-1
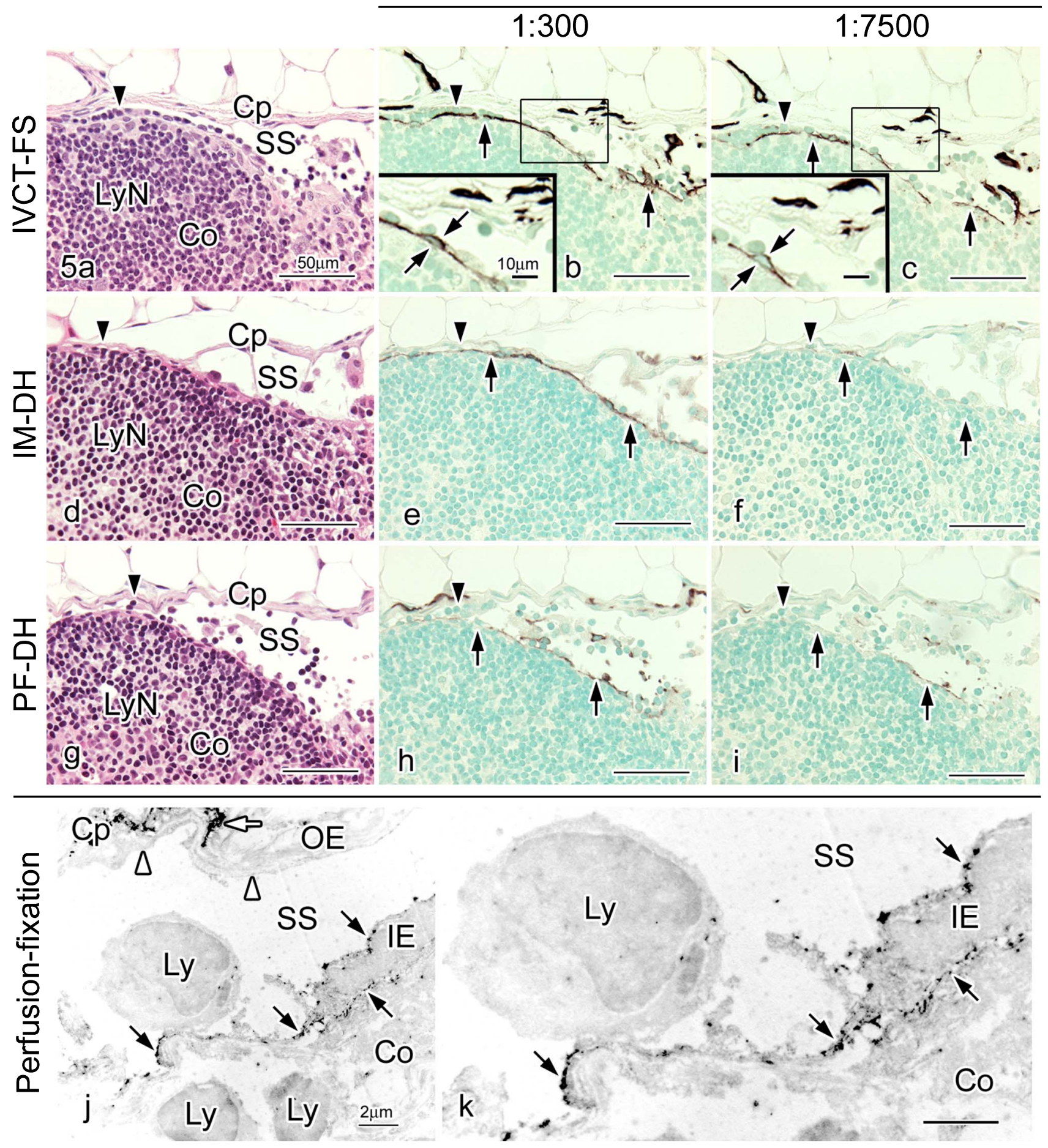

Fig. 5. Hematoxylin-eosin (HE) staining (a, d, g) and LYVE-1 immunostaining with different concentrations of primary anti-LYVE-1 antibody (1:300 in $\mathbf{b}, \mathbf{e}, \mathbf{h}$ and 1:7500 in $\mathbf{c}, \mathbf{f}, \mathbf{i})$ in serial paraffin sections prepared by IVCT-FS (a-c), immersion-fixation followed by alcohol-dehydration (IM-DH; d-f) or perfusion-fixation followed by alcohol-dehydration (PF-DH; $\mathbf{g}-\mathbf{- i})$. Insets in (b) and (c) are highly magnified views of inner endothelial cells shown by the squares. With IVCT-FS, a row of lymphocytes along subcapsular sinus (SS) is observed in this area (arrowhead in a), compared to IM-DH or PF-DH (arrowheads in $\mathbf{d}$, g). The LYVE-1 immunoreactivity is more clearly detected at a 1:7500 primary antibody dilution in samples with IVCT-FS (c), but it is decreased in samples with IM-DH (f) or PF-DH (i). (j) and (k) Pre-embedding immunoelectron microscopy for LYVE-1 in a mesenteric lymph node. The LYVE-1 is immunolocalized at both luminal and basal cell membrane surfaces of inner endothelial cells (IE; black arrows) along subcapsular sinus (SS), but not in outer endothelial cells (OE; white arrowheads). The LYVE-1 is also immunolocalized in some cells in the interstitium of capsules (Cp) surrounding SS (white arrow). Co, Cortex; $\mathrm{Cp}$, Capsule; LyN, Lymphatic nodule; Ly, Lymphocyte; $\mathrm{k}$, higher magnification of IE. Bars $=50 \mu \mathrm{m}(\mathbf{a}-\mathbf{i}), 10 \mu \mathrm{m}$ (insets of $\mathbf{b}, \mathbf{c}), 2 \mu \mathrm{m}(\mathbf{j}, \mathbf{k})$. 

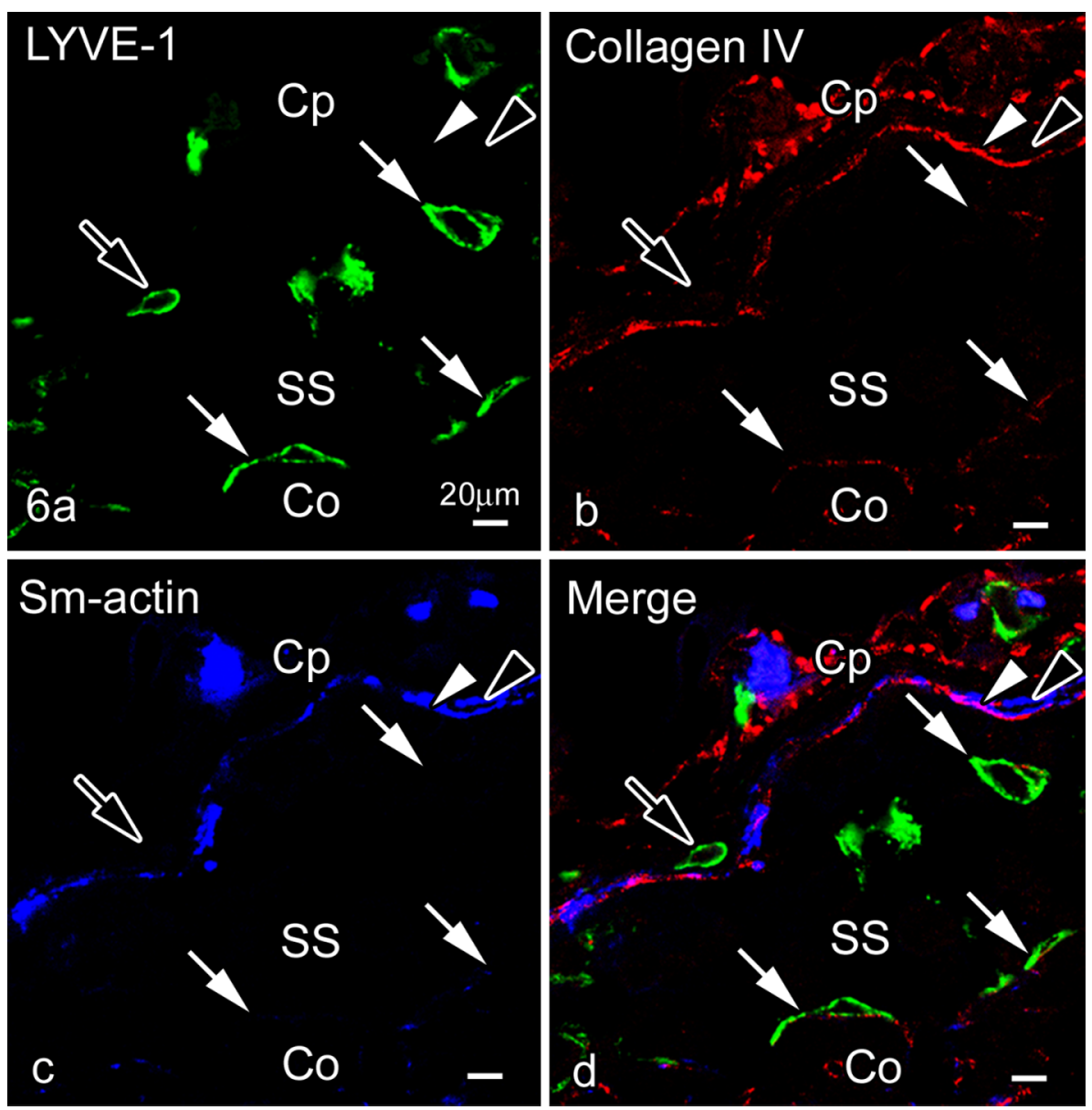

Fig. 6. Immunofluorescence labeling of LYVE-1 in cryosections of a mesenteric lymph node prepared by IVCTFS. Triple-immunoflurescence staining for LYVE-1 (green in a, d), type IV collagen $\alpha 2$ chain (Collagen IV; red in b, d), and smooth muscle actin (Smactin; blue in $\mathbf{c}, \mathbf{d}$ ). Intensity of type IV collagen (white arrowheads in $\mathbf{b}, \mathbf{d}$ ) and Sm-actin (black arrowheads in $\mathbf{c}, \mathbf{d}$ ) immunoreactivities is relatively strong along outer sides of SS. LYVE-1immunopositive cells are observed in the capsular stroma (black arrows in ad). The LYVE-1 is immunolocalized in inner endothelial cells along SS and reticular cells (white arrows in $\mathbf{a}-\mathbf{d}$ ), where type IV collagen or Sm-actin is faintly immunostained. Bars $=20 \mu \mathrm{m}$ (a-d). sides of inner endothelial cells. However, they were not seen in outer endothelial cells (arrowheads, Fig. 5j). In the connective tissues of capsules, they were seen in some cells other than endothelial cells (white arrows, Fig. 5j).

\section{Immunolocalization of type IV collagen or smooth muscle actin with LYVE-1 immunopositive endothelial cells}

Finally, immunofluorescence labeling of LYVE-1 was performed in mesenteric lymph nodes in cryosections prepared with IVCT-FS (Fig. 6). After triple-immunostaining for LYVE-1, type IV collagen and smooth muscle (Sm)actin, LYVE-1 was immunolocalized in the inner endothelial cells and reticular cells of the subcapsular sinuses, but not in outer endothelial cells, which were surrounded by type IV collagen and Sm-actin (Fig. 6a-d). Thus, the LYVE-1 immunolocalization was reconfirmed in the inner endothelial cells of the subcapsular sinuses.

\section{Discussion}

Subcapsular sinuses of lymph nodes have been thought to play some important roles for filtering lymph fluid to transport and process invading antigens into their parenchyma, as well as both adhesion and migration of immune cells and also metastatic cancer cells. The present study demonstrated histological features of living mouse mesenteric lymph nodes with IVCT, such as widely open lymphatic sinuses and original locations of flowing lymphocytes. It is also interesting to note that many small lymphocytes were attached to inner endothelial cells in subcapsular sinuses, as shown in Figures $2 \mathrm{~b}$ and $5 \mathrm{a}$. Thus, as the wide lumen of lymphatic sinuses and flowing lymphocytes were maintained by using IVCT, it may reflect the natural subcapsular sinuses of functional lymph nodes. Round fenestrations of endothelial cells were demonstrated at the parenchymal sides of the subcapsular sinus walls [35, 42, 43], indicating that the inner endothelial cells are used as a route for antigens and/or free cells to pass from the lymphatic sinuses to the parenchyma of lymph nodes. As IVCT has been used to avoid various technical artifacts, such as shrinkage and distortion always caused by conventional preparation methods $[1,4,14,15,16,21,31,48]$, other dynamic experiments with fluorescent-marker injection are also under consideration [37].

The LYVE-1 was immunolocalized in the inner endothelial cells and reticular cells of subcapsular sinuses, but not in outer ones or in afferent lymphatic vessels, as shown in Figures 3-6. Immunoelectron microscopy also showed LYVE-1 in both luminal and basal cell membrane surfaces of inner endothelial cells, but immunoreactivity of LYVE-1 was strongly observed in the luminal sides than that of inner sides, as shown in Figure 5. The immunolocalization 


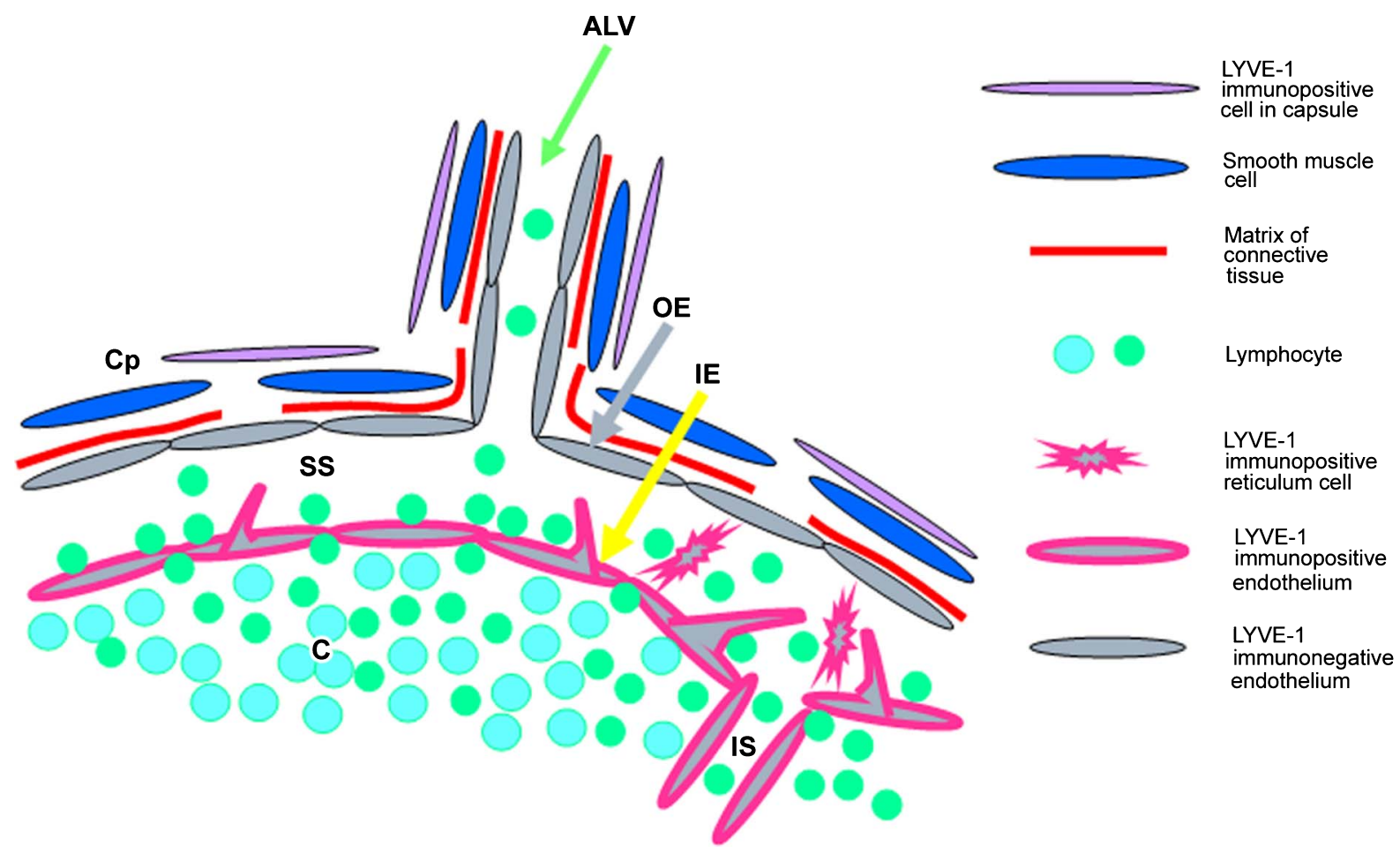

Fig. 7. Schematic representation of LYVE-1 immunolocalization in the subcapsular sinus (SS) of mouse mesenteric lymph nodes. LYVE-1 (pink) is localized in inner endothelial cells (IE) (yellow arrow) and reticulum cells of the SS or intermediate sinus (IS), but not in outer endothelial cells (OE) (grey arrow) and endothelial cells for afferent lymphatic vessel (ALV). Many lymphocytes (green) are attached to the IE. Matrices (red line) and smooth muscle cells (blue) in capsules (Cp) are organized under the basal side of the OE.

of LYVE-1 was first identified as a lymph-specific receptor for hyaluronic acid (HA) [2]. In addition, rather than facilitating degradation of HA, it was thought to be involved in HA transportation across lymphatic endothelial cells, specifically movement of accumulated HA from lymphatic sinuses to the lymph node parenchyma [29]. The possible roles of the lymphatic endothelial cells in HA homeostasis are also modified with HA binding by sialylation and selfassociation $[10,17]$. In addition, it was already shown that injected fluorophore-conjugated HA was taken through the endothelial cells of subcapsular sinuses [33]. The flow of lymphatic fluid was examined by injection of fluorophoreconjugated gelatin into the peritoneal cavity [24], showing its movement through endothelial cells to medullary labyrinths. But in the previous studies by using injection of soluble substances with different molecular weights, the lymphatic parenchyma was impenetrable to those molecules with about $70 \mathrm{kD}$ [7]. The IVCT could be used to keep the morphology in situ of lymph nodes, and dynamic experiments with fluorescence-labeled tracers will be possible to clarify it.

Our findings that the LYVE-1 was present at both luminal and basal surfaces of inner endothelial cells, and that the immunolocalization of LYVE-1 is more strongly observed in the apical sides of inner endothelial cells, as shown in Figure 5, are well compatible with this functional concept. As schematically summarized as a model in
Figure 7, LYVE-1 immunolocalization is different between inner and outer endothelial cells of subcapsular sinuses, probably reflecting the different functions for the transportation of HA and also the adhesion or migration of CD44-positive lymphocytes [8-10]. Such different features between inner and outer endothelial cells were already reported by another histochemical approach for hydrolytic enzymes [6].

Furthermore, as shown in Figure 6, LYVE-1 was not expressed in outer endothelial cells contacting with type IV collagen and/or smooth muscle cells. This finding is also consistent with a study of developing lymph vessels, in which mature lymphatic vessels surrounded by smooth muscle cells usually reduced the LYVE-1 expression [36]. Finally, as shown in Figures 3, 4 and 5, the LYVE-1 was immunolocalized in some cells of the capsules. As it was already reported to be expressed in some types of macrophages [5, 32, 47] and other cells [11, 12], identification of such cell types in the capsules will be another topic for future studies.

In conclusion, functioning tissue structures of mesenteric lymph nodes of living mice, probably reflecting their living states, were clearly described by the IVCT-FS method, and their LYVE-1 immunolocalization in the subcapsular sinus was also visualized in the inner endothelial cells, not in the outer ones, as summarized in Figure 7. 


\section{References}

1. Bai, Y., Ohno, N., Terada, N., Saitoh, S., Nakazawa, T., Nakamura, N., Katoh, R. and Ohno, S. (2009) Immunolocalization of serum proteins in xenografted mouse model of human tumor cells by various cryotechniques. Histol. Histopathol. 24; 717-728.

2. Banerji, S., Ni, J., Wang, S. X., Clasper, S., Su, J., Tammi, R., Jones, M. and Jackson, D. G. (1999) LYVE-1, a new homologue of the CD44 glycoprotein, is a lymph-specific receptor for hyaluronan. J. Cell Biol. 144; 789-801.

3. Breiteneder-Geleff, S., Soleiman, A., Kowalski, H., Horvat, R., Amann, G., Kriehuber, E., Diem, K., Weninger, W., Tschachler, E., Alitalo, A. and Kerjaschki, D. (1999) Angiosarcomas express mixed endothelial phenotypes of blood and lymphatic capillaries. Podoplanin as a specific marker for lymphatic endothelium. Am. J. Pathol. 154; 385-394.

4. Chan, F. L. and Inoue, S. (1994) Lamina lucida of basement membrane: an artefact. Microsc. Res. Tech. 28; 48-59.

5. Cho, C. H., Koh, Y. J., Han, J., Sung, H. K., Jong, L. H., Morisada, T., Schwendener, R. A., Brekken, R. A., Kang, G., Oike, Y., Choi, T. S., Suda, T., Yoo, O. J. and Koh, G. Y. (2007) Angiogenic role of LYVE-1-positive macrophages in adipose tissue. Circ. Res. 100; e47-57.

6. Crocker, J. and Williams, M. (1984) An enzyme histochemical study of the sinuses of reactive lymph nodes. J. Pathol. 142; 3138 .

7. Gretz, J. E., Norbury, C. C., Anderson, A. O., Proudfoot, A. E. and Shaw, S. (2000) Lymph-borne chemokines and other low molecular weight molecules reach high endothelial venules via specialized conduits while a functional barrier limits access to the lymphocyte microenvironments in lymph node cortex. J. Exp. Med. 192; 1425-1440.

8. Jackson, D. G., Prevo, R., Clasper, S. and Banerji, S. (2001) LYVE-1, the lymphatic system and tumor lymphangiogenesis. Trends Immunol. 22; 317-321.

9. Jackson, D. G. (2003) The lymphatics revisited: new perspectives from the hyaluronan receptor LYVE-1. Trends Cardiovasc. Med. $13 ; 1-7$.

10. Jackson, D. G. (2009) Immunological functions of hyaluronan and its receptors in the lymphatics. Immunol. Rev. 230; 216-231.

11. Jiang, S., Bailey, A. S., Goldman, D. C., Swain, J. R., Wong, M. H., Streeter, P. R. and Fleming, W. H. (2008) Hematopoietic stem cells contribute to lymphatic endothelium. PLOS ONE 3; e3812.

12. Junt, T., Moseman, E. A., Iannacone, M., Massberg, S., Lang, P. A., Boes, M., Fink, K., Henrickson, S. E., Shayakhmetov, D. M., Di Paolo, N. C., van Rooijen, N., Mempel, T. R., Whelan, S. P. and von Andrian, U. H. (2007) Subcapsular sinus macrophages in lymph nodes clear lymph-borne viruses and present them to antiviral B cells. Nature 450; 110-114.

13. Kaipainen, A., Korhonen, J., Mustonen, T., van Hinsbergh, V. W., Fang, G. H., Dumont, D., Breitman, M. and Alitalo, K. (1995) Expression of the fms-like tyrosine kinase 4 gene becomes restricted to lymphatic endothelium during development. Proc. Natl. Acad. Sci. U S A 92; 3566-3570.

14. Leu, F. J., Chen, C. F. and Sun, A. M. (1993) A new method of tissue processing that causes no shrinkage or distortion. Lab. Invest. 69; 121-130

15. Li, Z., Terada, N., Ohno, N. and Ohno, S. (2005) Immunohistochemical analyses on albumin and immunoglobulin in acute hypertensive mouse kidneys by "in vivo cryotechnique". Histol. Histopathol. 20; 807-816

16. Li, Z., Ohno, N., Terada, N. and Ohno, S. (2006) Immunolocalization of serum proteins in living mouse glomeruli under various hemodynamic conditions by "in vivo cryotechnique". Histochem. Cell Biol. 126; 399-406.

17. Nightingale, T. D., Frayne, M. E., Clasper, S., Banerji, S. and
Jackson, D. G. (2009) A mechanism of sialylation functionally silences the hyaluronan receptor LYVE-1 in lymphatic endothelium. J. Biol. Chem. 284; 3935-3945.

18. Ohno, N., Terada, N., Fujii, Y., Baba, T. and Ohno, S. (2004) "In vivo cryotechnique" for paradigm shift to "living morphology" of animal organs. Biomed. Rev. 15; 1-19.

19. Ohno, N., Terada, N. and Ohno, S. (2004) Advanced application of the in vivo cryotechnique to immunohistochemistry for animal organs. Acta Histochem. Cytochem. 37; 357-364

20. Ohno, N., Terada, N., Murata, S., Katoh, R. and Ohno, S. (2005) Application of cryotechniques with freeze-substitution for the immunohistochemical demonstration of intranuclear pCREB and chromosome territory. J. Histochem. Cytochem. 53; 55-62.

21. Ohno, N., Terada, N. and Ohno, S. (2006) Histochemical analyses of living mouse liver under different hemodynamic conditions by "in vivo cryotechque". Histochem. Cell. Biol. 126; 389-398.

22. Ohno, N., Terada, N., Bai, Y., Saitoh, S., Nakazawa, T., Nakamura, N., Naito, I., Katoh, R. and Ohno, S. (2008) Application of cryobiopsy to morphological and immunohistochemical analyses of xenografted human lung cancer tissues and functional blood vessels. Cancer 113; 1068-1079.

23. Ohno, S., Terada, N., Fujii, Y., Ueda, H. and Takayama, I. (1996) Dynamic structure of glomerular capillary loop as revealed by an in vivo cryotechnique. Virchows Arch. 427; 519-527.

24. Ohtani, O., Ohtani, Y., Carati, C. J. and Gannon, B. J. (2003) Fluid and cellular pathways of rat lymph nodes in relation to lymphatic labyrinths and Aquaporin-1 expression. Arch. Histol. Cytol. 66; 261-272.

25. Ohtani, O. and Ohtani, Y. (2008) Structure and function of rat lymph nodes. Arch. Histol. Cytol. 71; 69-76.

26. Petrova, T. V., Mäkinen, T., Mäkelä, T. P., Saarela, J., Virtanen, I., Ferrell, R. E., Finegold, D. N., Kerjaschki, D., Ylä-Herttuala, S. and Alitalo, K. (2002) Lymphatic endothelial reprogramming of vascular endothelial cells by the Prox-1 homeobox transcription factor. EMBO J. 21; 4593-4599.

27. Pfeiffer, F., Kumar, V., Butz, S., Vestweber, D., Imhof, B. A., Stein, J. V. and Engelhardt, B. (2008) Distinct molecular composition of blood and lymphatic vascular endothelial cell junctions establishes specific functional barriers within the peripheral lymph node. Eur. J. Immunol. 38; 2142-2155.

28. Phan, T. G., Grigorova, I., Okada, T. and Cyster, J. G. (2007) Subcapsular encounter and complement-dependent transport of immune complexes by lymph node B cells. Nat. Immunol. 8; 992-1000.

29. Prevo, R., Banerji, S., Ferguson, D. J., Clasper, S. and Jackson, D. G. (2001) Mouse LYVE-1 is an endocytic receptor for hyaluronan in lymphatic endothelium. J. Biol. Chem. 276; 19420-19430.

30. Sado, Y., Kagawa, M., Kishiro, Y., Sugihara, K., Naito, I., Seyer, J. M., Sugimoto, M., Oohashi, T. and Ninomiya, Y. (1995) Establishment by the rat lymph node method of epitope-defined monoclonal antibodies recognizing the six different $\alpha$ chains of human type IV collagen. Histochem. Cell Biol. 104; 267-275.

31. Saitoh, S., Terada, N., Ohno, N. and Ohno, S. (2008) Distribution of immunoglobulin-producing cells in immunized mouse spleens revealed with "in vivo cryotechnique". J. Immunol. Methods 29; 114-126.

32. Schledzewski, K., Falkowski, M., Moldenhauer, G., Metharom, P., Kzhyshkowska, J., Ganss, R., Demory, A., FalkowskaHansen, B., Kurzen, H., Ugurel, S., Geginat, G., Arnold, B. and Goerdt, S. (2006) Lymphatic endothelium-specific hyaluronan receptor LYVE-1 is expressed by stabilin-1+, F4/80+, CD11b+ macrophages in malignant tumours and wound healing tissue in vivo and in bone marrow cultures in vitro: implications for the assessment of lymphangiogenesis. J. Pathol. 209; 67-77.

33. Sharma, R., Wang, W., Rasmussen, J. C., Joshi, A., Houston, J. P., Adams, K. E., Cameron, A., Ke, S., Kwon, S., Mawad, M. E. and Sevick-Muraca, E. M. (2007) Quantitative imaging of lymph 
function. Am. J. Physiol. Heart Circ. Physiol. 292; 109-118.

34. Shimo, S., Saitoh, S., Terada, N., Ohno, N., Saitoh, Y. and Ohno, S. (2010) Immunohistochemical detection of soluble immunoglobulins in living mouse small intestines using an in vivo cryotechnique. J. Immunol. Methods 361; 64-74.

35. Szakal, A. K., Holmes, K. L. and Tew, J. G. (1983) Transport of immune complexes from the subcapsular sinus to lymph node follicles on the surface of nonphagocytic cells, including cells with dendritic morphology. J. Immunol. 131; 1714-1727.

36. Tammela, T., Saaristo, A., Holopainen, T., Lyytikkä, J., Kotronen, A., Pitkonen, M., Abo-Ramadan, U., Ylä-Herttuala, S., Petrova T. V. and Alitalo, K. (2007) Therapeutic differentiation and maturation of lymphatic vessels after lymph node dissection and transplantation. Nat. Med. 13; 1458-1466.

37. Terada, N., Ohno, N., Li, Z., Fujii, Y., Baba, T. and Ohno, S. (2005) Detection of injected fluorescence-conjugated $\mathrm{IgG}$ in living mouse organs using "in vivo cryotechnique" with freezesubstitution. Microsc. Res. Tech. 66; 173-178.

38. Terada, N., Ohno, N., Li, Z., Fujii, Y., Baba, T. and Ohno, S. (2006) Application of in vivo cryotechnique to the examination of cells and tissues in living animal organs. Histol. Histopathol. $21 ; 265-272$

39. Terada, N., Ohno, N., Saitoh, S., Saitoh, Y. and Ohno, S. (2009) Immunoreactivity of glutamate in mouse retina inner segment of photoreceptors with in vivo cryotechnique. J. Histochem. Cytochem. 57; 883-888.

40. Terada, N., Ohno, N., Saitoh, S., Saitoh, Y., Fujii, Y., Kondo, T., Katoh, R., Chan, C., Abraham, S. N. and Ohno, S. (2009) Involvement of dynamin-2 in formation of discoid vesicles in urinary bladder umbrella cells. Cell Tissue Res. 337; 91-102.

41. Terada, N., Ohno, N., Saitoh, S., Saitoh, Y., Komada, H., Kubota,
H. and Ohno, S. (2010) Involvement of a membrane skeletal protein, 4.1G, for Sertoli/germ cell interaction. Reproduction 139; 883-892.

42. Ushiki, T., Ohtani, O. and Abe, K. (1995) Scanning electron microscopic studies of reticular framework in the rat mesenteric lymph node. Anat. Rec. 241; 113-122.

43. van Rooijen, N. (1987) The "in situ" immune response in lymph nodes: a review. Anat. Rec. 218; 359-364.

44. Ward, J. M., Erexson, C. R., Faucette, L. J., Foley, J. F., Dijkstra, C. and Cattoretti, G. (2006) Immunohistochemical markers for the rodent immune system. Toxicol. Pathol. 34; 616-630.

45. Wetterwald, A., Hoffstetter, W., Cecchini, M. G., Lanske, B., Wagner, C., Fleisch, H. and Atkinson, M. (1996) Characterization and cloning of the E11 antigen, a marker expressed by rat osteoblasts and osteocytes. Bone 18; 125-132.

46. Wigle, J. T. and Oliver, G. (1999) Prox 1 function is required for the development of the murine lymphatic system. Cell 98; 769778 .

47. Xu, H., Chen, M., Reid, D. M. and Forrester, J. V. (2007) LYVE1-positive macrophages are present in normal murine eyes. Invest. Ophthalmol. Vis. Sci. 48; 2162-2171.

48. Zhou, D., Ohno, N., Terada, N., Li, Z., Morita, H., Inui, K., Yoshimura, A. and Ohno, S. (2007) Immunohistochemical analyses on serum proteins in nephrons of protein-overload mice by "in vivo cryotechnique". Histol. Histopathol. 22; 137-145.

This is an open access article distributed under the Creative Commons Attribution License, which permits unrestricted use, distribution, and reproduction in any medium, provided the original work is properly cited 